\title{
Religion und Zeit in Christoph Ransmayrs Roman Cox oder Der Lauf der Zeit
}

Ein Beitrag zum Diskurs über die Religion in der Literatur der Gegenwart

Religion et temps dans le roman Cox oder Der Lauf der Zeit de Christoph

Ransmayr. Une contribution au discours sur la religion dans la littérature

contemporaine.

Religion and Time in Christoph Ransmayr's Novel Cox oder der Lauf der Zeit.

A Contribution to the Discourse on Religion in Contemporary Literature

\section{Sikander Singh}

\section{(2) OpenEdition}

Journals

Édition électronique

URL : http://journals.openedition.org/ceg/3172

DOI : $10.4000 /$ ceg. 3172

ISSN : 2605-8359

Éditeur

Presses Universitaires de Provence

Édition imprimée

Date de publication : 26 avril 2018

Pagination : 151-162

ISBN : 979-10-320-0160-8

ISSN : 0751-4239

Référence électronique

Sikander Singh, "Religion und Zeit in Christoph Ransmayrs Roman Cox oder Der Lauf der Zeit », Cahiers d'Études Germaniques [Online], 74 | 2018, Online erschienen am: 26 Oktober 2019, abgerufen am 26 November 2020. URL : http://journals.openedition.org/ceg/3172 ; DOI : https://doi.org/10.4000/ceg. 3172 


\title{
Religion und Zeit in Christoph Ransmayrs Roman Cox oder Der Lauf der Zeit
}

\author{
Ein Beitrag zum Diskurs über die Religion in der \\ Literatur der Gegenwart
}

\author{
Sikander Singh \\ Universität des Saarlandes, Saarbrücken
}

\section{Aspekte der Religion und Literatur seit der Moderne}

In den Fragmenten, die Friedrich von Hardenberg 1798 an Friedrich Schlegel zur Veröffentlichung in der Zeitschrift Athenaeum übersandte, in der Blüthenstaub überschriebenen Sammlung, die unter einem Titel veröffentlicht wurde, der das Unabgeschlossen-Werdende und doch bereits Vollendete des romantischen Weltentwurfs programmatisch darstellt, steht auch eine Reflexion über Ursprung und Wesen der Literatur: „Dichter und Priester waren im Anfang Eins, und nur spätere Zeiten haben sie getrennt", schreibt Novalis und fährt fort, der „ächte Dichter ist aber immer Priester, so wie der ächte Priester immer Dichter geblieben. Und sollte nicht die Zukunft den alten Zustand der Dinge wieder herbeyführen?" ${ }^{1}$ Jenseits der Selbststilisierung, die den Dichter in den Bereich des Kultus entrückt, der dem Priester - dem in die letzten Geheimnisse Eingeweihten - vorbehalten ist, behauptet Novalis, indem er die Geschichte der Dichtung implizit als einen Prozess der Säkularisierung begreift, eine der Kunst immanente transzendente Dimension.

In die von Johann Peter Eckermann und Friedrich Wilhelm Riemer unter dem Titel Maximen und Reflexionen zusammengestellten Aphorismen und Sentenzen wurde eine aus dem Nachlass überlieferte Betrachtung aufgenommen, in der Johann Wolfgang Goethe ebenfalls über das Verhältnis der Literatur zur Religion nachdenkt: „Religion: Alte; / Poesie: Religion der Jugend.“2 Der nur knapp umrissene Gedanke, der nicht in die syntaktische Einheit eines Satzes gebracht, sondern in einer Weise kontrapunktisch festgehalten worden ist, die

1. August Wilhelm Schlegel / Friedrich Schlegel, Athenaeum. Eine Zeitschrift, Berlin, Vieweg, 1798, Bd. 1, S. 90.

2. Goethes Werke, hrsg. im Auftrage der Großherzogin Sophie von Sachsen, Weimar, Böhlau, 1887-1919, 1. Abt., Bd. 42 / 2, S. 213. 
den Prozess des Denkens, dem er entstammt, sichtbar macht, kann einerseits als Ausdruck einer autobiographischen Nachdenklichkeit verstanden werden: In dem sich verdichtenden Bewusstsein der eigenen Sterblichkeit denkt Goethe über die letzten Fragen nach und anerkennt - sich selbst mit einer lebensklugen Ironie beobachtend - die Notwendigkeit einer Religion im Angesicht des Todes. Andererseits kann seine Reflexion als eine kritische Betrachtung über das emphatische Heilsversprechen gelesen werden, das die frühe Romantik in der Dichtung zu erkennen glaubte.

Novalis' Fragment und Goethes Reflexion dokumentieren exemplarisch, mit welchen Hypostasierungen und Argumenten seit dem aufgeklärten 18. Jahrhundert über das Erbe der christlichen Tradition gestritten worden ist. Die Literatur hat einen wesentlichen Anteil an diesen Debatten, denn in der Nachfolge von Vorstellungen, die von der Frühromantik formuliert worden sind, entfaltete sich bis zum 21. Jahrhundert eine Deutung der literarischen Moderne als Produkt der fortschreitenden Verweltlichung der Rede von und über die Religion. ${ }^{3}$

Dass Religion und religiöser Diskurs die Literatur gleichwohl bis in die Gegenwart bestimmen, zeigt sich in erzählerischen Modellen, Strukturen und Sprechweisen, aber auch in Motiven, Stoffen, Figuren und intertextuellen Bezugnahmen auf religiöses Schrifttum, die als Sediment wie als Ferment dichterischen Schreibens anzusehen sind. ${ }^{4}$ Diese „kontinuierliche[...] Präsenz der Religion“ ${ }^{5}$ verläuft zwar mit den Säkularisierungsprozessen parallel, die das Religiöse seit der Aufklärung aus dem Feld gesellschaftlicher, sozialer und politischer Relevanz in den Bereich intellektueller Reflexion abgedrängt haben. Sie ist jedoch weder Konsequenz noch Ergebnis eines historischen Fortschritts aus dem Geist des Rationalismus oder Materialismus - wie dies von der idealistischen Geschichtsphilosophie behauptet wird -, vielmehr dokumentiert sie lediglich den Prozess einer kulturellen Transformation und produktiven Verwandlung religiöser Themen und Denkfiguren. Weil diese aus den tradierten sakralen Kontexten herausgelöst werden, entstehen neue Formen des religiösspirituellen Diskurses und mit ihnen neue Möglichkeiten der Reflexion über die Grenzen der Immanenz und der Spekulation über die Transzendenz.

Der vorliegende Beitrag, der den Roman Cox oder Der Lauf der Zeit von Christoph Ransmayr einer genauen Lektüre im Sinne eines close reading unterzieht, arbeitet vor diesem Hintergrund die religiösen Ideen und

3. Vgl. Dorothee Sölle, Realisation. Studien zum Verhältnis von Theologie und Dichtung nach der Aufklärung, Darmstadt / Neuwied, Luchterhand, 1973.

4. Vgl. u. a. Albrecht Grözinger, Andreas Mauz, Adrian Portmann (Hrsg.), Religion und Gegenwartsliteratur. Spielarten einer Liaison, Würzburg, Königshausen \&t Neumann, 2009 [Interpretation Interdisziplinär 6]; Tim Lörke, Robert Walter-Hochum (Hrsg.), Religion und Literatur im 20. und 21. Jahrhundert. Motive, Sprechweise, Medien, Göttingen, V \& R unipress, 2015; Klaus von Stosch, Sabine Schmitz, Michael Hofmann (Hrsg.), Kultur und Religion. Eine interdisziplinäre Bestandsaufnahme, Bielefeld, transcript, 2016.

5. Clemens Pornschlegel, Fromme Ignoranz. Überlegungen zum Verhältnis von Literatur und Religion in der Moderne, in Michael Hofmann, Klaus von Stosch (Hrsg.), Islam in der deutschen und türkischen Literatur, Paderborn / München / Wien / Zürich, Schöningh, 2012 [Beiträge zur komparativen Theologie 4], S. 95-107, hier S. 96. 
Repräsentationen heraus, die das Werk, das zu der Frankfurter Buchmesse des Jahres 2016 ausgeliefert wurde, wesentlich bestimmen. Ausgehend von der These, dass der österreichische Schriftsteller jene romantische Denkfigur aufnimmt und fortschreibt, der zufolge der Kunst eine sakrale Dimension innewohnt, wird die literarische Bildlichkeit des Romans auf ihren religiösen Gehalt befragt und im Kontext der literarischen Traditionslinien, denen sie verpflichtet ist, gedeutet.

\section{Literarische Form - gedanklicher Gehalt}

In einem Brief schreibt Ossip Mandelstam 1931 über sein Werk Die Reise nach Armenien: „Mein Buch spricht davon, daß das Auge ein Instrument des Denkens ist, daß das Licht eine Kraft, und daß das Ornament Gedanke ist." Diesen triadischen Ansatz zu einer Selbstdeutung vertiefend fügt er hinzu: „Es handelt von Freundschaft, von Wissenschaft, von intellektueller Leidenschaft, und nicht von ,Dingen“." ${ }^{6}$ Die Selbstreflexion des Dichters, die auf das Verhältnis von philosophischem Gehalt und ästhetischer Form, gedanklicher Konzeption und Wirkungsabsicht seiner Reisebeschreibung aufmerksam macht, kann auch auf das jüngste Werk Ransmayrs bezogen werden.

Der Roman Cox oder Der Lauf der Zeit erzählt die Geschichte eines britischen Uhrmachers und Automatenkonstrukteurs. Alister Cox ist ein Mann, der alles verloren hat, was er liebte. Sein Kind ist eines frühen Todes gestorben und seine Ehefrau hat sich ihm in der Folge dieser Verlusterfahrung entzogen - ist ihm unerreichbar geworden und hat ihn allein dem Schmerz und der Trauer überlassen. Indem er nichts mehr zu verlieren hat, folgt er der Einladung eines Fürsten in ein fernes Land: Begleitet von drei Gefährten, ebenfalls Uhrmachern, reist er nach China, um am kaiserlichen Hof mechanische Zeitmesser zu fertigen, kunstvoll erdachte Uhrwerke, welche die Leidenschaft des Herrschers für die handwerkliche Kunst der Europäer befriedigen sollen.

Der Roman beginnt mit der Ankunft des Uhrmachers in der kaiserlichen Residenzstadt, erzählt von der Pracht und dem Reichtum der Hofhaltung, von dem die Gäste faszinierenden wie verstörenden Zeremoniell, den Ver- und Geboten, von grausamen Urteilen und ihrem Vollzug, von Beamten und Höflingen, welche die Arbeit der Ausländer zunächst skeptisch, zunehmend mit Feindschaft betrachten, von den seltenen und ungewöhnlichen Begegnungen mit dem Kaiser Quiánlóng, schließlich von seinen Wünschen, wie die Befehle von seinem Umfeld genannt werden. Zunächst erbittet er den Bau einer Uhr, welche „die fliegenden, kriechenden oder erstarrten Zeiten eines menschlichen Lebens“ anzuzeigen im Stande wäre, ein Maß für „das wechselnde Tempo der Zeit“" ${ }^{7}$. Wenngleich die Konstruktion eines solchen Instruments Cox vor keine Herausforderung stellt, wird die Arbeit an dem Werk zu einer Möglichkeit, über das eigene Leben, seine

6. Ossip Mandelstam, Die Reise nach Armenien, Frankfurt a. M., Suhrkamp, 1997 [Bibliothek Suhrkamp 801], S. 7.

7. Christoph Ransmayr, Cox oder Der Lauf der Zeit. Roman, Frankfurt a. M., Fischer, 2016, S. 83. 
Wechselläufe und Verluste nachzudenken. Noch bevor der Auftrag ausgeführt ist, formuliert der Kaiser einen anderen Wunsch. Die Engländer sollen eine „Uhr für Todgeweihte“ fertigen, einen „Zeitmesser für zum Tode Verurteilte und alle, die das Datum ihres Todes kannten, das Ende ihres Lebens unabweisbar kommen sahen und sich nicht mehr mit der Hoffnung auf eine Art dehnbarer, vorläufiger Unsterblichkeit besänftigen durften“" ${ }^{8}$. Auch hierfür stehen alle Reichtümer und Möglichkeiten des kaiserlichen Hofes zur Verfügung.

Aber auch dieses Uhrwerk wird, trotz des gedanklichen wie materiellen Aufwandes, der zu seiner Realisation betrieben wird, nicht vollendet, denn Quiánlóng erteilt schließlich den Auftrag, eine Uhr zu bauen, „die über alle Menschenzeit in den Sternenraum hinausschlug, ohne jemals stillzustehen, und deren Grenzen allein in der Dauer und dem Geheimnis der Materie selbst lagen“9 . Der Meister ist zwar über die „Maßlosigkeit“ und die „Allmachtsansprüche[...]“ 10 des Kaisers erschrocken. Der Auftrag korrespondiert jedoch mit einer eigenen, im Verborgenen gehegten Wunschvorstellung, ein „Perpetuum mobile“"11 zu fertigen. So spiegelt sich in dem kaiserlichen Wunsch nach Macht zugleich der auf die Grenzen des technisch Möglichen gerichtete Ehrgeiz des Uhrmachers.

Das Vorhaben droht zwar an den Gesetzen der Physik wie an administrativen Widerständen zu scheitern; trotzdem können die Engländer am Ende ein mechanisches Werk übergeben, das den Lauf der Stunden durch einen sorgsam erdachten Antriebsmechanismus bis in die Ewigkeit zu messen verspricht. Für ihre geleisteten Dienste werden sie großzügig entlohnt und reisen ab, um in ihre Heimat zurückzukehren. Quiánlóng, dem es vorbehalten ist, den Mechanismus der Uhr in Gang zu setzen, verzichtet im Schlussbild auf diesen letzten Schritt.

Ransmayrs Erzähltext, der darüber nachdenkt, wie die „Träume eines Kaisers in Mechanik zu verwandeln" ${ }^{12}$ sein möchten, ist im Hinblick auf die Form ein Historischer Roman. Das Werk wie ein ihm beigegebenes Nachwort, das mit dem temporalen Adverb Zuletzt überschrieben ist, lassen keinen Zweifel daran, dass seine Handlung im 18. Jahrhundert, dem Zeitalter der Aufklärung, angesiedelt ist. Auch der Umstand, dass Cox ein Uhrmacher ist und damit an Voltaire erinnert, der in Ferney eine Taschenuhren-Manufaktur betrieb, macht den Roman zu einer literarischen Betrachtung über die in und nach der Aufklärung diskutierte Frage nach Möglichkeiten wie Grenzen menschlicher Vernunft. ${ }^{13}$ Aber der Uhrmacher und der Kaiser, die Automatensammlung in der Verbotenen Stadt, die Schauplätze, die Haupt- und Nebenfiguren des Romans sind, trotz ihrer Korrespondenz mit historischen Vorbildern fiktiv, oder, wie der Autor in der Nachschrift formuliert, eine „Erfindung“"14.

8. Ibid., S. 106.

9. Ibid., S. 213.

10. Ibid., S. 214.

11. Ibid., S. 274.

12. Ibid., S. 192.

13. Vgl. u. a. Pierre Lepape, Voltaire oder die Geburt der Intellektuellen im Zeitalter der Aufklärung, Frankfurt a. M., Campus, 1996.

14. Ransmayr, Cox, S. 303. 
Dass der Text nicht an der Rekonstruktion des Faktischen interessiert ist, zeigt sich auch in der Struktur der Erzählung, die Phantastisches und Reales, Erdachtes und Historisches miteinander verschränkt und so den Kunstcharakter des Werkes betont. Sie zeigt sich zudem in der Erzählung von der Anfertigung eines mechanischen Instruments, das im Widerspruch zu den „unvergänglichen Gesetze[n] der Physik“ ${ }^{15}$ steht, in der Realisation eines zeitmessenden Mechanismus, der deshalb unmöglich ist.

Indem der Historische Roman (im Gegensatz zu der Tradition der Gattung) nicht die Wirklichkeit seines Gegenstandes behauptet, sondern sein eigenes Narrativ textimmanent als fiktional markiert, scheint zwar der seit der Poetik des Aristoteles geführte Diskurs über das Verhältnis von literarischem und historischem Erzählen auf. Der Text lenkt das Erkenntnisinteresse jedoch einerseits auf die gedankliche Triade, die Mandelstam im Hinblick auf die Deutung seiner armenischen Reise formuliert. Andererseits entsteht mit den Bildern der mechanischen Zeitmesser, die Cox entwirft, und mit den Betrachtungen, die der Uhrmacher, der chinesische Kaiser wie der Erzähler über die Zeit und ihren Lauf anstellen, eine dichterische Reflexion über das menschliche Leben, seine Endlichkeit und sein Verhältnis zu zeitlichen Kategorien, die sowohl über den Horizont individueller Existenz als auch über die Möglichkeiten der Vorstellungskraft hinausweisen. Die Uhren werden in diesem Sinne als „helle, funkelnde Gleichnisse und Vorahnungen der Ewigkeit" 16 charakterisiert. Die Ewigkeit wird, in einer bestürzend materialistischen Denkfigur, als eine Zeit verstanden, „in der es keine Zeit und keine Ziele mehr“ ${ }^{17}$ gibt.

Zugleich macht der Roman durch seine formale Anlage die Aporie sichtbar, die in jedem Versuch einer Konzeptualisierung von Zeit liegt: Cox erzählt weder von einer definitiven Vergangenheit noch von einer bestimmbaren Gegenwart. Indem er Figuren und Namen mit der „Wirklichkeit“ lediglich „teilt“ und damit eine anachronistische Distanz bewahrt, vollzieht der Text eine erzählerische Bewegung außerhalb der Linearität der Zeit. Wie die Skeptiker der antiken Philosophie, die eine gesicherte Erkenntnis über die Welt für unmöglich erachteten und deshalb die Aporie als den Endpunkt jeglichen philosophischen Bemühens verstanden, sind Gegenstand und Form des Romans auf die Einsicht in die Unmöglichkeit ausgerichtet, zu der Auflösung des behandelten Problems zu gelangen. Der Historische Roman, der von einer Vergangenheit erzählt, die es nicht gegeben hat, wiederholt daher allein durch seine Form die Aporie, in die jedes Nachdenken über die Kategorie der Zeit führen muss.

Diese Spiegelung des inhaltlich reflektierten Problems in der Form ist auch deshalb schlüssig, weil sie mit der Idee des Uhrmachers korrespondiert, es könnte möglich sein, „ein mechanisches Abbild seiner [der kaiserlichen] Macht über die Zeit“ ${ }^{18}$ zu schaffen. Die Uhr „schlug ihre Stunden über alle Grenzen des Tages

15. Ibid., S. 219.

16. Ibid., S. 112.

17. Ibid., S. 190.

18. Ibid., S. 254. 
und der Jahre hinaus und brauchte dabei niemanden, der ihren Gang nach der Erschöpfung aller Reserven um weitere Umlaufperioden verlängerte“ ${ }^{19}$, heißt es im 16. Kapitel. Der Roman thematisiert so die Möglichkeit, in der Fiktion das sichtbar und damit auch wirklich werden zu lassen, was den Gesetzen der Realität entgegenläuft.

Die Einheit von gedanklichem Gehalt und literarischer Form ist deshalb als Metonymie zu verstehen. Der Text zeigt eine Relation im Sinne einer Kontiguität zwischen der physikalischen Größe der Zeit, deren Fluss der Mensch mit mechanischen Instrumenten messbar zu machen und damit zu konzeptualisieren sucht, und dem letzten Grund im Sinne der Transzendenz. Die Zeit, die zu messen und $\mathrm{zu}$ verstehen die Figuren des Romans auf je eigene Weise den Versuch unternehmen, ist eine Chiffre für das Überschreiten der endlichen, menschlichen Erfahrungswelt. In einer Uhr, „die ihre Stunden aus dem Inneren der Zeit in die Zeitlosigkeit schlug “20, wie der Erzähler räsoniert, wird dies in besonderer Weise sinnfällig, ebenso in den Figuren und deren Betrachtungen sowie den behutsam in den Text integrierten Kommentierungen des Erzählers.

So erscheint der chinesische Kaiser in einer ambivalenten Perspektive. Einleitend betont der Erzähler, dass Cox in ein „verheißungsvolles, von einem Gottkaiser beherrschtes Land“ ${ }^{21}$ reist. Auch sein Beiname „Wàn suì yé: Herr der zehntausend Jahre“ ${ }^{22}$ hebt das Sakrale hervor, das mit ihm in der chinesischen Theokratie wesenhaft verbunden ist. In seiner Person fallen diesseitige und jenseitige Macht in eins. Die Verehrung als „Gottgleiche[r]“ ${ }^{23}$ gipfelt schließlich in der Auffassung seines Reiches als „ein Abbild des Himmels“24. Und in der Deutung seiner Sommerresidenz Jehol, die nach den Entwürfen und Plänen des Kaisers errichtet worden ist und die sein Hofstaat (mit ihnen auch die britischen Uhrmacher) in jedem Sommer bezieht, als „das vom imperialen Willen zur Erde herabbefohlene Universum“25.

Die Cognomina können zwar als Chiffren für Machtfülle und -vollkommenheit des Monarchen gelesen werden; auch bestätigt das Geschehen immer wieder, dass Quiánlóng „der mächtigste Mann der Welt“26 ist. Zugleich erheben die Deutungen den Kaiser über den Bereich des Menschlichen. Nicht nur seine Person ist absolut, die Verehrung, die ihm entgegengebracht wird, ist es ebenfalls. Seine Apotheose kontrastiert allerdings mit den Bildern, die Cox aufgrund eigener Beobachtungen gewinnt und die Darstellung und - damit verbunden - Wertung des Herrschers bestimmen.

Letzterer ist ihm, aufgrund der Berichte und Andeutungen des Übersetzers Joseph Kiang, der die Engländer stets begleitet, zunächst eine geradezu irreal-

19. Ibid., S. 269.

20. Ibid., S. 241.

21. Ibid., S. 18.

22. Ibid., S. 61.

23. Ibid., S. 26.

24. Ibid., S. 30.

25. Ibid., S. 199.

26. Ibid., S. 9. 
imaginäre Gestalt. In dem 5. Kapitel, das von der ersten Audienz berichtet, heißt es deshalb einleitend: „Schließlich war es eine Sache, von der Macht eines Menschen, der über Leben und Tod bestimmen konnte, ohne jemals von einem Einspruch behindert werden zu können, nur zu wissen - und eine andere, diesem Menschen gegenüberzutreten und vor ihm auf die Knie zu sinken." ${ }^{27}$ Nach der Begegnung, während derer Cox „die Gedanken des Kaisers von China als die Gedanken eines einfachen Mannes lesen konnte“ - schließlich handelt das Gespräch „Vom Lauf der Zeit und von Uhren“ -, erkennt er jedoch, dass „auch der gottgleiche Kaiser von China - ein Mensch“" ${ }^{28}$ ist. Die relativierende Einsicht, die durch die Kapitelüberschrift Shi jiān, ein Mensch zusätzlich hervorgehoben wird, bestimmt nicht nur die weiteren Begegnungen. Weil sie die Perspektive der Beamten und Höflinge in Frage stellt, diskutiert der Text die Fragwürdigkeit aller Macht von Menschen über Menschen - eine Frage, die bereits in Ransmayrs Roman Die letzte Welt ein zentrales Thema war. In dem 1988 veröffentlichten Werk wird der Dichter Ovid von Kaiser Augustus aus Rom, dem Zentrum des römischen Reiches, an die Küste des Schwarzen Meeres verbannt, weil seine Dichtungen die uneingeschränkte Macht des Imperators in Frage zu stellen scheinen. Cox schreibt also die Betrachtung über das Verhältnis von Kunst und politischer Herrschaft, die Ransmayr in der Letzten Welt begonnen hat, fort. ${ }^{29}$

Das Bild, das der Erzähler von dem „Herr[n] der zehntausend Jahre“, der „nicht nur über Anfang und Ende der Zeit, sondern auch über ihre Messung und das Tempo ihres Vergehens gebot“" ${ }^{30}$, zeichnet, wird zu einem Sinnbild für die Vergänglichkeit alles Menschlichen gesteigert. Denn im weiteren Verlauf der Handlung werden die Engländer in der kaiserlichen Sommerresidenz zu einer Audienz an den Fluss bestellt. Dort sitzt der „mächtigste Mann der Welt ohne das geringste Zeichen seines Ranges“, er ist „in einen wassergrauen, bleigrauen Umhang gehüllt“ und gleicht einem „Hirte[n] oder Fischer“31. Der Kaiser von China ist kein „gottähnlicher, unvergleichlicher Herrscher, sondern nur [...] einer von vielen“32. Das Bild des Flusses, dessen fortwährender, ununterbrochener Strom das Vergehen der Zeit sichtbar macht und so an die Vergänglichkeit des Seienden mahnt, relativiert alle irdische Macht, hebt die „Unterschiede zwischen den Menschen“ auf - auch die „zwischen der organischen und anorganischen Natur“, zwischen ,jedem Ding und jedem Wesen“33.

Am Ende dieser Begegnung steht deshalb ein Erzählkommentar, der, in eine rhetorische Frage gekleidet, ein Thema aufnimmt, das Ransmayr wiederholt beschäftigt hat: Die fortgesetzte Verwandlung alles Seienden, die Flüchtigkeit

27. Ibid., S. 70 .

28. Ibid., S. 84.

29. Vgl. hierzu u. a. Swenta Steinig, Postmoderne Phantasien über Macht und Ohnmacht der Kunst. Vergleichende Betrachtung von Süskinds „Parfüm“ und Ransmayrs „Letzte Welt“, Literatur für Leser 1, 1997, S. 37-51.

30. Ransmayr, Cox, S. 205.

31. Ibid., S. 210.

32. Ibid., S. $210 f$.

33. Ibid., S. 211. 
aller Form und die existentielle Ratlosigkeit des Menschen, der zu der Einsicht in das Unbedingte und jedes Verstehen Übersteigende dieser Prozesse gelangt - weshalb die offen bleibende Frage die einzig angemessene literarische Form hierfür ist:

\begin{abstract}
Was blieb schließlich selbst von einem Stern, einer von Planeten, Asteroiden, Monden und Meteoriten umschwärmten Sonne, deren Licht vor Jahrmilliarden entflammt war? Und was von allen anderen, noch in kommenden Äonen aufgehenden Himmelslichtern, die im unerbittlichen Lauf dieser Zeit allesamt wieder zu einem Schwarm von namenlosen Partikeln zerspringen würden, atomaren Bausteinen, die in einer unbegreiflichen Zukunft unter dem Druck von Gewalten jenseits aller Vorstellungskraft aufs Neue zu elementaren Formationen verklumpen konnten, um sich allmählich, rotierend, wachsend zu Gestalten von nie gesehener Größe, nie gesehener Schönheit oder Häßlichkeit aufzublähen ... Und dies alles nur, um nach Ablauf ihrer Daseinsfrist wiederum zur Unsichtbarkeit in tiefster Finsternis zu zerfallen ${ }^{34}$
\end{abstract}

Die parallel verlaufenden, einander bedingenden und kommentierenden Gedanken des Uhrmachers und des Erzählers zeichnen das nach, für das der Text in der Geschichte von dem Bau einer „Uhr für die Ewigkeit“35, eines Mechanismus, der unabhängig von den physikalischen Wechselwirkungen ist, ein dinghaftes Symbol liefert. Das Instrument, das erkennen nicht nur seine Konstrukteure sondern auch die Mächtigen am Hof, stellt bereits durch seine bloße Existenz die Herrschaft und die Autorität des „Herr[n] der zehntausend Jahre“ ${ }^{36}$ in Frage - und damit auch die von ihm repräsentierte Ordnung, durch die sich die Beamten und Höflinge wiederum selbst ermächtigt sehen.

Der Hofstaat beginnt die Uhr, „eine Maschine, die den Weg aus aller Ordnung in die Zeitlosigkeit weisen sollte“" ${ }^{37}$, nicht nur zu hassen, weil der Kaiser für ihre Vollendung alle ihm zur Verfügung stehenden Ressourcen bereitstellt. In der Perspektivierung menschlicher Existenz auf die Ewigkeit verliert die Gegenwart, verlieren Machtstrukturen und Ordnungsmuster ihre bindende Kraft, dominiert stattdessen eine demütige Haltung aus dem Bewusstsein des Zurückbleibens hinter einer opaken Vollkommenheit jenseits des Menschlichen.

Cox strebt nicht nach dem Höchsten und Vollkommensten, das für menschlichen Geist und feinmechanisches Geschick erreichbar ist, nicht das drängende Suchen im Sinne der Volkssage von Doktor Faust ist sein Ziel. Zwar spiegelt sich auch in den Gesprächen zwischen ihm und seinem Gefährten Jacob Merlin ein Moment menschlicher Hybris, eine Erhebung und Selbstermächtigung aus dem Glauben an die Möglichkeiten des technischen Fortschritts. Sein Denken wird vor allem von der Erfahrung der Vergänglichkeit, die durch den Tod seiner geliebten Tochter in sein Bewusstsein getreten ist, bestimmt. Indem die Arbeit an Maschinen, die den Lauf der Zeit messen, eine Mediation über den erlittenen Verlust ist, wird sie auch zu einem Trost. Über diesen Aspekt, der das Faustische mit einem Moment subjektiver Erfahrung kontrastiert, gelangt Cox

\footnotetext{
34. Ibid., S. $211 \mathrm{f}$.

35. Ibid., S. 217.

36. Ibid., S. 61.

37. Ibid., S. 233.
} 
zu der Erkenntnis, dass der Bau einer Uhr, die „keine Menschen mehr braucht“ ${ }^{38}$ auch die Macht des Kaisers grundlegend in Frage stellt:

Und eine Uhr [...], die diesen Kaiser überragen sollte, sich über seine Tage hinaus drehte, und auch ihn am Ende als bloßen Statisten eines übergeordneten Zeitenlaufs erscheinen ließ, mußte doch wohl mit dem Anspruch verbunden sein, dauerhafter, größer zu sein als er selbst! Dauerhafter als der Herr über die Zeit, der dadurch zu einem Menschen, einem von vielen, schrumpfte. Und alles, worüber er gebot, was er besaß, was ihn erfreute oder was er liebte, verwandelte sich durch diesen Zauber zum wertlosen Treibgut [...]. ${ }^{39}$

Der Wunsch des Kaisers ist daher auch Sinnbild für die Selbstüberhebung des Menschen. Indem der Roman die Möglichkeit eröffnet, eine Wunschvorstellung in der Welt des Textes Wirklichkeit werden zu lassen, vermag der Erzähltext das Unbedeutende menschlichen Strebens zu veranschaulichen. Die Erkenntnis der Relativität wird jedoch nicht nur durch den Fortgang der Erzählung für den Leser fassbar. Während der „Herr der zehntausend Jahre“40 in der letzten Szene des Romans allein vor der Maschine steht, die durch seinen Willen Wirklichkeit geworden ist, wird auch ihm bewusst, dass eine mechanische Schöpfung, die den Horizont ihrer Schöpfer überragt, seine eigene Position nicht erhöht, sondern relativiert:

\begin{abstract}
Aber wenn er nun dieses weiter und weiter und weiter schlagende Werk in Gang setze, wurde der Lauf der Zeit dann nicht unbezweifelbar, für alle Geborenen und noch die Ungeborenen fernster Epochen ablesbar von seinem Fächer aus Skalen - und unwiderruflich? Und konnte ein Herr über zehntausend Jahre dann noch allein nach seinem Willen über die Zeit verfügen oder driftete er in ihrem Fluß wie irgendeiner seiner namenlosen Untertanen dahin? ${ }^{41}$
\end{abstract}

\title{
Immanenz - Transzendenz
}

Cox oder Der Lauf der Zeit untersucht die Möglichkeiten der metaphorischen Sprechweise der Literatur im Hinblick auf das Verhältnis des Menschen zu den letzten Dingen. Der Roman steht damit auch in der Tradition der romantischen Vorstellungen, welche die transzendente Dimension der Dichtung als wesenhaftes Moment derselben behaupteten. Während diese Deutungen der Literatur bis in das 20. Jahrhundert auf die paradigmatische Stellung der christlichen Überlieferung bezogen blieben, die Novalis und seine Weggefährten programmatisch betonten, zeigt Ransmayr im beginnenden 21. Jahrhundert die Substitution der christlichen Gottesvorstellung und Eschatologie durch eine Reflexion über die Transzendenz. Die Differenz zwischen den Betrachtungen und Fragen, die Cox behandelt, und den religiösen Traditionen Europas wird in dem Roman nicht nur durch die räumliche Entfernung des Geschehens versinnbildlicht. Die Ablösung von der christlichen Überlieferung wird auch benannt: So verweist der Erzähler im 8. Kapitel, die

\footnotetext{
38. Ibid., S. 257.

39. Ibid., S. 241.

40. Ibid., S. 61.

41. Ibid., S. 298.
} 
Erzählzeit raffend, darauf, dass die „Wochen des Hochwinters verstrichen“ und dass „Qiánlóngs Reich“ nicht „die geringste Notiz von der Geburt eines Gottes in einem staubigen, heiligen Land" 42 nahm. Indem die Bedingtheit religiöser Traditionen erzählerisch veranschaulicht wird, zeigt der Roman den Menschen als Suchenden, der fragend der existenziellen Seinserfahrung begegnet.

Bereits Die Schrecken des Eises und der Finsternis, die von einer Nordpolarexpedition in den Jahren 1872 bis 1874 handeln, erzählt von einer Suche: der Suche nach der Nordostpassage. Die letzte Welt zeichnet die vergebliche Suche des Römers Cotta nach dem verschwundenen Dichter Ovid und dessen letztem Werk nach. Und Der fliegende Berg berichtet von dem Aufstieg zweier Brüder zu einem unbekannten Gipfel im Himalaya-Gebirge, ein Gang, der zu einer Suche nach der eigenen Vergangenheit wird. In Cox ist die Suche nicht nur Handlungsmodell, vielmehr thematisiert der Text die Frage nach dem menschlichen Seinsverständnis und der Bezogenheit seines Denkens auf das Metaphysische. Die Uhr, „die jede Stunde des Lebens und Todes ihrer Erbauer und ihrer Nachkommen bis in die fernste Zukunft ohne jedes weitere menschliche Zutun messen und anzeigen konnte" 43 , ist eine Chiffre für jenes Begehren nach einem sich stets entziehenden Ursprung und Zusammenhang des Sinns, das Jacques Lacan und ihm nachfolgend Jacques Derrida als metaphysisch beschrieben haben ${ }^{44}$. Obwohl die Zeit mit Hilfe von mechanischen Geräten als messbare Größe physikalisch konzeptualisierbar wird, ist sie in ihrem Wesen unbestimmbar. Weil ihre Qualität wie Quantität dem räumlichen Paradigma entzogen sind, bleibt sie abstrakt, hermetisch. Sie ist - und darin gleicht sie der Idee eines sie bis zum Ende aller Zeiten messenden Instruments - „für menschliche Hände so unerreichbar [...] wie der Thron eines Gottes“45.

Cox lässt seine „Manufakturen in Liverpool, London und Manchester“46 hinter sich, um nach einer sechsmonatigen Schiffspassage in einem fremden Land Uhren zu fertigen und damit jene Tätigkeit wieder aufzunehmen, die er zurückgelassen hat. Die Einladung des chinesischen Kaisers ist der bloße Anlass seiner Reise; ihr Grund ist der Tod seiner Tochter. Der Uhrmacher ist, wie der Erzähler herausstellt, „der traurigste[...] Mann der Welt“" ${ }^{47}$, sein Weg in die Ferne ist ein Versuch, für das Ungeklärte und nunmehr Haltlose des eigenen Daseins einen Ort zu finden. Der Roman schließt so auch an Walter Benjamins Essay Der Erzähler an, der davon spricht, dass der „Gedanke der Ewigkeit“ von ,jeher seine stärkste Quelle im Tod gehabt“" ${ }^{48}$ habe.

42. Ibid., S. 118.

43. Ibid., S. 274.

44. Vgl. hierzu u. a. Jacques Lacan, Das Seminar. Buch 7. Die Ethik der Psychoanalyse, Weinheim / Berlin, Quadriga 1996.

45. Ransmayr, Cox, S. 274.

46. Ibid., S. 22.

47. Ibid.

48. Walter Benjamin, Der Erzähler. Betrachtungen zum Werk Nikolai Lesskows, in ders., Gesammelte Schriften, hrsg. von Rolf Tiedemann, Hermann Schweppenhäuser, Frankfurt a. M., Suhrkamp, 1972-1999, Bd. 2 / 2, S. 449. 
Indem die Reise des Uhrmachers auf den Tod eines Kindes bezogen ist, und indem die Arbeit an feinmechanischen Instrumenten die Frage nach dem Sinn menschlichen Strebens stellt, erweist sich der Roman als eine literarische Betrachtung über das Leben, die Sterblichkeit, die Zeit sowie ihr Verhältnis zueinander. ${ }^{49}$ Während Die letzte Welt über das Unerbittliche „einer alles vernichtenden, alles verwandelnden Vergänglichkeit“50 nachdenkt, reflektiert Cox die Verletzlichkeit und Endlichkeit menschlicher Existenz. Das Erkenntnisinteresse, das in den frühen Romanen auf das Verschwinden alles Seienden, die Erosion allen Sinns gerichtet ist, nimmt in Ransmayrs neuem Roman die Bedeutung des Transzendenten für den Menschen in den Blick. In der Frage, ob es die Zeit ist, die das Sein „in eine Sequenz überführt“ und ihm „Sinn verleiht“ ${ }^{51}$, liegt eine Suchbewegung, die aus der Immanenz auf die Transzendenz hindeutet. Der Text, seine Figuren und sein Handlungsgefüge denken vom Tode her; sie sind auf jene unvorstellbare Vorstellung gerichtet, die mit jedem Begriff nur unzureichend und mit keinem auch nur annähernd benannt werden kann.

Ransmayr wählt für diese Suchbewegung eines über den Urgrund seiner Existenz nachdenkenden Menschen die erzählerische Form. Aber nicht nur das Geschehen, die Betrachtungen der Figuren, die Kommentare des Erzählers denken über das Transzendente und Absolute nach. Dem Roman ist als literarischer Gattung, wie Benjamin in seinem Essay herausarbeitet, eine Bewegung zum Transzendenten als integraler Bestandteil bereits eingeschrieben:

\footnotetext{
Nicht darum also ist der Roman bedeutend, weil er, etwa lehrreich, ein fremdes Schicksal uns darstellt, sondern weil dieses fremde Schicksal kraft der Flamme, von der es verzehrt wird, die Wärme an uns abgibt, die wir aus unserem eigenen nie gewinnen. Das was den Leser zum Roman zieht, ist die Hoffnung, sein fröstelndes Leben an einem Tod, von dem er liest, zu wärmen. ${ }^{52}$
}

Weil die Stimme des Erzählers und der mehrstimmige Chor seiner Figuren ihre Autorität dem Absoluten des Todes (also einer Kategorie ohne Zeit) entliehen haben, verweist der Roman als Sequenz, als eine Abfolge von Handlungen auf das Endliche. ${ }^{53}$ Cox ist deshalb der modellhafte Versuch, die „Grenzen der Wirklichkeit“" ${ }^{54}$ mit den Mitteln des Erzählers zu erkunden. Damit ist der Roman auch als ein Experiment zu lesen; der Erzähler, der im Bereich des Erzählten das Verhältnis des Historischen und des Erdachten sowie die Erzählgeschwindigkeit bestimmt, ist als ein Herrscher über die Zeit zu deuten. Seine Gewalt über die Vergangenheit, die Gegenwart, die Zukunft oder ihre Verschränkung sind

49. Seiner Tochter „Lebensuhr hatte Cox den unauffälligen, je nach Jahreszeit von Blüten, Laub oder Hagebutten getarnten Grabschmuck genannt, an dem er das Vergehen seines eigenen Lebens ablesen und an Abigails ewige Ruhe binden wollte“ (Ransmayr, Cox, S. 22).

50. Christoph Ransmayr, Die letzte Welt. Roman. Mit einem Ovidischen Repertoire, Nördlingen, Greno, 1988 [Die Andere Bibliothek, hrsg. von Hans Magnus Enzensberger], S. 93.

51. Ali Smith, Wem erzähle ich das?, München, Luchterhand, 2017, S. 32.

52. Benjamin, Der Erzähler. Betrachtungen zum Werk Nikolai Lesskows, Bd. 2 / 2, S. $456 f$.

53. „Der Tod ist die Sanktion von allem, was der Erzähler berichten kann. Vom Tode her hat er seine Autorität geliehen“ (ibid., S. 450).

54. Ransmayr, Cox, S. 229. 
zwar weitaus weniger begrenzt als die Macht, die der „Herr der zehntausend Jahre“ 55 über die Zeit hat. Indem diese in der nachgestellten Betrachtung als eine „Erfindung“ ${ }^{56}$ benannt und damit fiktionsimmanent als fiktiv markiert wird, untersucht der Text das Verhältnis von Wirklichkeit und Literatur. Als ein Roman über den Roman spürt Ransmayrs Text den „Grenzen der Wirklichkeit“ ${ }^{57}$ nach.

Das Werk zielt zwar nicht darauf, eine Vorstellung dessen zu erzeugen, was hinter der Welt und ihren Erscheinungen liegen könnte. In der erzählenden Auseinandersetzung mit der Zeit scheint jedoch die Möglichkeit auf, dem Opaken, jedem Begreifen Entzogenen einen Ausdruck zu geben. In dem Roman gerinnt die Suche nach Spiritualität in einem säkularen, nicht der Religion, aber ihren traditionellen Vorstellungen entfremdeten Zeitalter deshalb in einer Chiffre. Zugrunde liegt dieser Denkfigur eine Vertauschung, die transnominatio der traditionellen Benennung Gottes in eine messbare, aber gleichwohl abstrakte, in eine vertraute, aber trotzdem dem Verstehen sich enthebende Kategorie - die Zeit.

In dem Sinne Ossip Mandelstams handelt der Roman nicht von „Dingen“, von Automaten, mechanischen Instrumenten, von Manufakturen oder Sammlungen. Indem die Geschichte eines Feinmechanikers und seiner Begegnung mit dem Kaiser von China erzählt wird, ist das Werk die Geschichte einer Freundschaft, einer Beziehung zweier Menschen, die einander fern sind und bleiben, aus dem ungeheuren Abstand ihrer Welten aber gleichwohl miteinander verwandt sind. „Konnten also dieser Kaiser und dieser englische Uhrmacher über Ozeane, Sprachräume und Denksysteme hinweg durch so etwas wie Seelenverwandtschaft verbunden sein?“ ${ }^{58}$, formuliert das 13 . Kapitel in eine rhetorische Frage gekleidet. Das Verbindende dieser beiden ist in dem Sinne einer intellektuellen Leidenschaft das Streben nach dem, von dem der Mensch nichts wissen kann:

Dieser seltsam zerbrechlich erscheinende und dabei schrankenlos mächtige Mensch, welche Kostüme und unaussprechlichen Titel er auch tragen mochte, träumte offensichtlich von ähnlichen Dingen wie er [Cox] und Merlin, träumte von einer Uhr, deren Räderwerk sich in eine Zukunft ohne Grenzen und Maß drehte. ${ }^{59}$

Wie bereits in Die letzte Welt verweist das Phantastische, das Anachronistische und Hybride, das dem Roman eingeschrieben ist, verweisen religiöser und philosophischer, wissenschaftlicher und dichterischer Diskurs, die einander durchdringen, auf die letzten Fragen. ${ }^{60}$ „Diese Dinge sind niemals geschehen, aber es gibt sie immer"61, schreibt der spätantike Philosoph Salustios, ein Neuplatoniker, in seiner Abhandlung Über die Götter und die Welt.

55. Ibid., S. 61.

56. Ibid., S. 303.

57. Ibid., S. 229.

58. Ibid., S. 217.

59. Ibid., S. $217 \mathrm{f}$.

60. Vgl. Anne-Marie Monluçon, "Hybride et métamorphose d'Ovide à Christoph Ransmayr ", in Ovide, figures de l'hybride. Illustrations littéraires et figurées de l'esthétique ovidienne à travers les âges, hrsg. von Hélène Casanova-Robin, Paris, Champion, 2009, S. 461-472.

61. Smith, Wem erzähle ich das?, S. 47. 